\title{
MEASUREMENTS OF WOMEN'S AUTONOMY IN REPRODUCTIVE HEALTH IN DEVELOPING COUNTRIES: A LITERATURE REVIEW
}

\author{
Yunita Amraeni ${ }^{1 *}$, M. Nirwan ${ }^{2}$ \\ 1,2 Universitas Mandala Waluya \\ Kendari, Southeast Sulawesi, Indonesia
}

Corresponding Author : Yunita Amraeni

Email : zahra.kahfi@gmail.com

\section{Abstract}

Background: The autonomy associated with the essence of decision-making in the field of reproductive health, including about fertility, pregnancy, and the utilization of health services. The research has shown that autonomy occurred in poor countries and growing. This literature is intended to explore autonomy measures and policies related to reproductive health programs. Methods: Review of the literature search some databases such as the Online Public Access Catalog (OPAC) and Pubmed Medical Center (PMC). Twenty-two articles that met the criteria for discussion included articles dominated by South and Southeast Asia and parts of Africa, as well as one European region. Most of the literature defines women's autonomy using theories from previous literature.

Results: the study proves that there is a link between autonomy and utilization of health services, family planning and fertility. Autonomy measurement is done by using direct and indirect dimensions. Dimensions direct connect participation in decision making related to the economy, household and mobility. The other dimension is to assess women's attitudes toward domestic violence. Dimensions are indirectly related to proxies that affect women's status such as employment, education or media exposure.

Conclusion: Potential policies and programs related to reproductive health in developing countries basically recommend the integration of women's empowerment in health programs.

Key words: Women, Autonomy, Health, Reproduction, Developing, Countries. 


\section{INTRODUCTION}

The reproductive health rights become one of the international agenda in 1994, the International Conference of Population Development (ICPD) conference in Cairo emphasis about humanism approach and human rights in looking at population and development issues and on the role of empowering women in reproductive behavior. The reproductive health also become a priority program to improve the women roles7,23.

Autonomy assessment is an indicator in increasing the role of women16. Dason and Moore (1983) explain autonomy as a person's ability to participate in the environment, be able to access information freely and be involved in decision making. Married women have increased autonomy so they can make decisions in the household and have power over personal rights such as household affairs and financial arrangements 28,36 .

Socio-economic and social is one dimension of autonomy. Countries with patriarchal ideas such as Bangladesh, India, including Indonesia have the same problem in autonomy. This understanding puts men in a more powerful position than women. Haque's (2012) study in Bangladesh and Widyastuti (2017) in Indonesia show that autonomy affects women's decisions in using ANC. Likewise, research in India shows that there is a link between women's autonomy and the use of health services and the improvement of reproductive health 32 . Other studies have shown that women with higher status may control fertility and reproductive health25.

Gender dynamics related to sexual and reproductive health began to be encouraged after the ICPD Conference in 1994. Based on the facts, there are limitations for women to participate in decisions in the household and family environment 12. This continues to deepen the international agenda, MDG's 2015, SDG's, and the World Bank's world development report 2012, which places the main program on issues of women's rights and gender.

Based on the concept of Kabeer (1999; 2001) reproductive health is associated with women's autonomy. therefore, research is needed to support the theory by measuring autonomy. Specifically, the objectives of this study are 1) To identify the linkages between autonomy and various reproductive health outcomes, 2) to examine indicators of measuring autonomy with various reproductive health outcomes.

\section{METHODS}

Search Strategy and Study Inclusions Searches were initially carried out on all literature sites such as Pubmed, Elseiver, Bmj, Biomed, Science direct and others .However, there is no literature on the measurement of autonomy. The search for literature sources is done by electronic online databases with Online Public Access Catalog (OPAC) and Pubmed Medical Centre (PMC). The addition of sources of information is done using Google Scholar, and relevant articles are also used as references. The selection process is described in the Preferred Reporting Items for Systematic Reviews and Meta-analyses (PRISMA) framework in Figure 1. Inclusion criteria consist of 1) English and Indonesian 2) Assessing the links between autonomy and at least one of the reproductive health outcomes. 3)Describe clearly the measurement of autonomy 4)Describe associations, not opinions or literature 5) limitation of 2000-2020.The exclusion criteria were articles with the keywords above, however 1) targeting the general population (not married women) 2) did not address women's autonomy. 3) articles other than English and Indonesian.. 


\section{RESULTS}

Literature consists of 541 publications related topics literature search found only in Public Access Catalogue (OPAC), Pubmed Medical Centre (PMC) and Google Scholar. After article duplication selection, there were 2566 articles left. Then screening the relevant article titles and abstracts. Publications with primary and secondary data are included when discussing autonomy and one of the effects of reproductive health, so that the remaining 29 articles with full texts will be assessed and then 7 articles are released because they only contain reviews and opinions. And at the end of the search, 22 articles were included in the selection for review. The evaluation of the quality of articles was not carried out in this study because the purpose of the synthesis of the literature was to summarize the assessment of the effect size of the review article (Figure 1)

Most studies are conducted in parts of South Asia, ten in parts of Africa and two in parts of Southeast Asia and Europe. All studies used quantitative methods for data collection, dominated by secondary data with a Demographic Health Survey (DHS), the rest using primary data.

Autonomy and various effects of reproductive health

Most of the literature defines female autonomy using theory from previous literature. Another study combines the definition of female autonomy by Dyson and Basu as the capacity to manipulate the environment related to controlling resources and information for personal desires. Dominant studies discuss the relevance of autonomy with the use of reproductive health services, six articles discuss Family Planning, five publications suggesting pregnancy, and the rest link autonomy with fertility and sexual activity.

Autonomy measurement indicator
Measurement of autonomy is carried out using direct and indirect dimensions. The direct dimension links participation in decision making related to the economy, household and mobility. Another dimension is assessing women's attitudes towards domestic violence. The dimensions are indirectly related to proxies that affect women's status such as employment, education or media exposure. So that causes gender differences between men and women. Various literature uses these dimensions singly or in combination. Most of the literature uses dimensions of participation in decision making in households for measuring autonomy.

On a single basis, participation in the household was used in the research of Hindin (2011) and Rahman (2012) with the discussion of pregnancy. Another single dimension uses attitudes towards domestic violence by Woldemicael (2009) related to fertility. The combination of participation in the household and attitudes towards violence were used in several studies related to the utilization of health services (Upadhay\& Karasek,2012;Situ.K.C,2013;Tadesse et al,2013;samari Goleen,2017; Tiruneh et al,2017). Another combination is indicated by using dimensions of participation in households and mobility (Mistry et al,2009;Renuka\&Jeyarathman,2016;Haider,2 017). Then a combination of direct and indirect dimensions is also found in the literature (Adhikari\&Yothin,2011;Kamiya,2011). 
Amraeni, Y., \& Nirwan, M.

DOI: 10.36566/ijhsrd/Vol3.Iss2/89

https://ijhsrd.com/index.php/ijhsrd

e- ISSN: 2715-4718

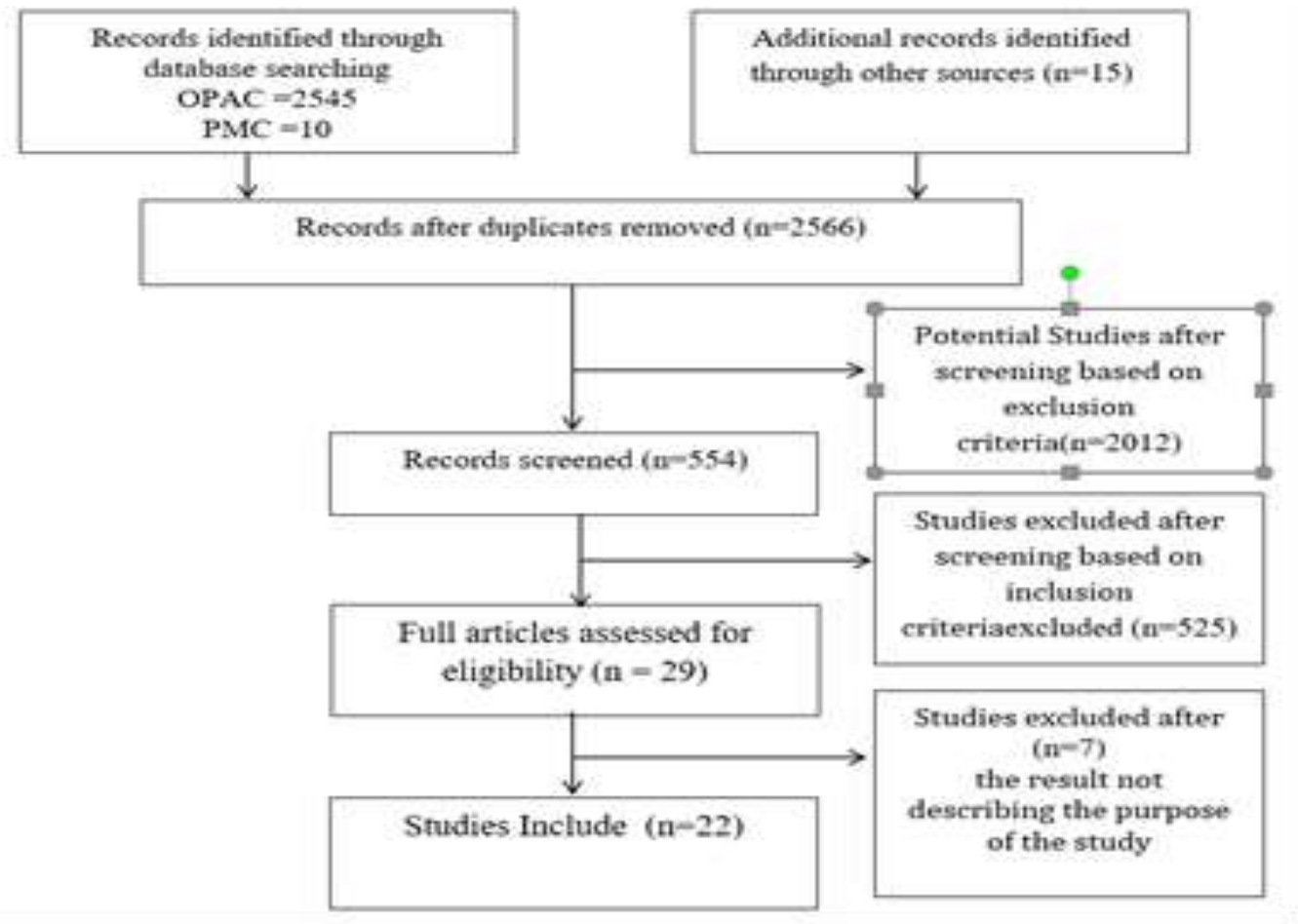

Figure 1.Flow of Systematic Review

Table. 1. Characteristics of Review Articles

\begin{tabular}{|c|c|c|c|c|c|c|}
\hline No & $\begin{array}{c}\text { Author/year of } \\
\text { publication }\end{array}$ & Setting & Study Objectives & $\begin{array}{l}\text { Study } \\
\text { Design }\end{array}$ & Sample size & $\begin{array}{c}\text { Data } \\
\text { Source }\end{array}$ \\
\hline 1 & $\begin{array}{l}\text { Upadhyay and } \\
\text { deborah/2012 }\end{array}$ & Afrika & $\begin{array}{l}\text { Understand whether women's } \\
\text { empowerment is associated with } \\
\text { their ideal number of children and } \\
\text { ability to limit fertility to that } \\
\text { ideal number }\end{array}$ & Survey & $\begin{array}{l}\mathrm{n}= \\
\text { matched } \\
\text { couples } \\
\text { Guinea, } \\
\mathrm{n}=\quad 2,668 \text { in } \\
\text { Mali, } 844 \text { in } \\
\text { Namibia and } \mathrm{n}= \\
\begin{array}{l}3,197 \\
\text { Zambia. }\end{array}\end{array}$ & $\begin{array}{l}2005,2006,2 \\
007 \text { DHS } \\
\text { survey }\end{array}$ \\
\hline 2 & $\begin{array}{l}\text { Tadesse et al/ } \\
\text { 2013. }\end{array}$ & $\begin{array}{l}\text { Afrika } \\
\text { (Ethiopi } \\
\text { a) }\end{array}$ & $\begin{array}{l}\text { Exploring major gender-related } \\
\text { factors that can potentially } \\
\text { influence the use of contraception } \\
\text { among women who are married } \\
\text { or living with a partner. }\end{array}$ & Survey & $\begin{array}{l}\mathrm{n}=10,204 \text { who } \\
\text { were currently } \\
\text { married }\end{array}$ & $\begin{array}{l}2011 \\
\text { Ethiopia } \\
\text { Demographi } \\
\text { c and Health } \\
\text { Survey } \\
\text { (EDHS) }\end{array}$ \\
\hline 3 & $\begin{array}{l}\text { Stojanovski } \\
\text { et.al/2017 }\end{array}$ & $\begin{array}{l}\text { Eropa(R } \\
\text { oma) }\end{array}$ & $\begin{array}{l}\text { Examine women's agency and } \\
\text { how it related to desired timing of } \\
\text { pregnancy among Romani women } \\
\text { in Macedonia and Serbia }\end{array}$ & Survey & $\begin{array}{l}\mathrm{n}=410 \text { Romani } \\
\text { women }\end{array}$ & $\begin{array}{l}\text { Primary } \\
\text { data }\end{array}$ \\
\hline 4 & $\begin{array}{l}\text { Goleen } \quad \text { Samari } \\
/ \mathbf{2 0 1 7}\end{array}$ & Mesir & $\begin{array}{l}\text { Examine recent patterns of } \\
\text { contraceptive method choice and } \\
\text { how women's empowerment is } \\
\text { associated with contraceptive } \\
\text { method type: none, short-acting } \\
\text { or long-acting reversible } \\
\text { contraceptive methods }\end{array}$ & Survey & $\begin{array}{l}\mathrm{n}=47,545 \\
\text { married women }\end{array}$ & $\begin{array}{l}2005,2008 \\
\text { and } 2014 \\
\text { Egyptian } \\
\text { Demographi } \\
\text { c and Health } \\
\text { Survey }\end{array}$ \\
\hline
\end{tabular}

Indonesian Journal Of Health Sciences Research and Development 
Amraeni, Y., \& Nirwan, M.

DOI: 10.36566/ijhsrd/Vol3.Iss2/89

https://ijhsrd.com/index.php/ijhsrd

e- ISSN: 2715-4718

\begin{tabular}{|c|c|c|c|c|c|c|}
\hline No & $\begin{array}{c}\text { Author/year of } \\
\text { publication }\end{array}$ & Setting & Study Objectives & $\begin{array}{c}\text { Study } \\
\text { Design }\end{array}$ & Sample size & $\begin{array}{c}\text { Data } \\
\text { Source }\end{array}$ \\
\hline 5 & $\begin{array}{l}\text { Renuka and } \\
\text { Jeyarathman/2016 }\end{array}$ & $\begin{array}{l}\text { Afrika } \\
\text { (Coimba } \\
\text { tore) }\end{array}$ & $\begin{array}{l}\text { This study was carried out to } \\
\text { identify the relationship between } \\
\text { women's autonomy and family } \\
\text { planning practices }\end{array}$ & Survey & $\begin{array}{l}\mathrm{n}=56 \text { married } \\
\text { women }\end{array}$ & $\begin{array}{l}\text { Primary } \\
\text { Data }\end{array}$ \\
\hline 6 & $\begin{array}{l}\text { Mosfequr } \\
\text { Rahman/ 2012 }\end{array}$ & $\begin{array}{l}\text { Banglad } \\
\text { esh }\end{array}$ & $\begin{array}{l}\text { Examines the net effect of } \\
\text { women's autonomy on their } \\
\text { pregnancy intention status among } \\
\text { currently pregnant Bangladeshi } \\
\text { women }\end{array}$ & Survey & $\begin{array}{ll}\mathrm{n}= & 718 \\
\text { currently } & \\
\text { pregnant } & \\
\text { women } & \end{array}$ & $\begin{array}{l}\text { Bangladesh } \\
\text { Demographi } \\
\text { c Health } \\
\text { Survey, } \\
2007\end{array}$ \\
\hline 7 & $\begin{array}{l}\text { Patrikar,et.al/ } \\
2014\end{array}$ & India & $\begin{array}{l}\text { Analyze the relationship between } \\
\text { these two indicators of women's } \\
\text { empowerment and the use of } \\
\text { contraception. }\end{array}$ & Survey & $\begin{array}{l}385 \text { currently } \\
\text { married women }\end{array}$ & $\begin{array}{l}\text { Primary } \\
\text { data }\end{array}$ \\
\hline 8 & $\begin{array}{l}\text { Abada \& } \\
\text { Tenkorang/ 2012 }\end{array}$ & Filifina & $\begin{array}{l}\text { Examined autonomy contributes } \\
\text { to unwanted and mistimed births } \\
\text { in the Philippines }\end{array}$ & Survey & $\begin{array}{l}\mathrm{n}=13,945 \\
\text { women }\end{array}$ & $\begin{array}{l}2003 \\
\text { Philippines } \\
\text { National } \\
\text { Demographi } \\
\text { c and Health } \\
\text { Survey } \\
\end{array}$ \\
\hline 9 & Blackstone/ 2017 & $\begin{array}{l}\text { Afrika } \\
\text { (Ghana) }\end{array}$ & $\begin{array}{l}\text { Investigate whether women's } \\
\text { empowerment and status in the } \\
\text { household were associated with } \\
\text { contraceptive use }\end{array}$ & Survey & $\begin{array}{l}\mathrm{n}=1828 \text { women } \\
\text { aged } 15-49\end{array}$ & $\begin{array}{l}2014 \text { Ghana } \\
\text { Demographi } \\
\text { c and Health } \\
\text { Survey }\end{array}$ \\
\hline 10 & Ghose et.al/ 2017 & $\begin{array}{l}\text { Banglad } \\
\text { esh }\end{array}$ & $\begin{array}{l}\text { Determine the association } \\
\text { between women's decision- } \\
\text { making power and utilisation of } \\
\text { maternal healthcare services } \\
\text { (MHS) among Bangladeshi } \\
\text { women }\end{array}$ & Survey & $\begin{array}{l}\mathrm{n}=17.989 \\
\text { households }\end{array}$ & $\begin{array}{l}2014 \\
\text { Bangladesh } \\
\text { Demographi } \\
\text { c and Health } \\
\text { Survey }\end{array}$ \\
\hline 11 & $\begin{array}{l}\text { Woldemicael/ } \\
\mathbf{2 0 0 7}\end{array}$ & $\begin{array}{l}\text { Ethiopia } \\
\text { And } \\
\text { Eritrea }\end{array}$ & $\begin{array}{l}\text { Different dimensions of women's } \\
\text { decision-making autonomy and } \\
\text { their relationship to maternal and } \\
\text { child health-care utilization are } \\
\text { investigated }\end{array}$ & Survey & $\begin{array}{l}\mathrm{n}=14.070 \\
\text { women age } 15- \\
49 \text { years }\end{array}$ & $\begin{array}{l}\text { Demographi } \\
\text { c and Health } \\
\text { Surveys } \\
\text { (DHS) } \\
\text { conducted } \\
\text { in Eritrea } \\
\text { and Ethiopia } \\
\text { in } 2002 \text { and } \\
2005\end{array}$ \\
\hline 12 & $\begin{array}{l}\text { Furuta } \\
\text { and Salway/2006, }\end{array}$ & Nepal & $\begin{array}{l}\text { How a woman's position within } \\
\text { her household may affect the } \\
\text { receipt of health care deserves } \\
\text { further investigation }\end{array}$ & Survey & $\begin{array}{l}\mathrm{n}=8,400 \text { ever- } \\
\text { married women } \\
\text { aged } \\
15-49\end{array}$ & $\begin{array}{l}2001 \text { Nepal } \\
\text { Demographi } \\
\text { c and Health } \\
\text { Survey }\end{array}$ \\
\hline 13 & Mistry et,al/ 2009 & India & $\begin{array}{l}\text { Investigate whether women's } \\
\text { autonomy was associated with the } \\
\text { use of adequate prenatal, delivery } \\
\text { and postnatal care }\end{array}$ & Survey & $\begin{array}{l}\mathrm{n}=90,303 \\
\text { women age } 15- \\
49 \text { years }\end{array}$ & $\begin{array}{l}\text { Population- } \\
\text { based } \\
\text { National } \\
\text { Family } \\
\text { Health } \\
\text { Survey-2 } \\
(1998- \\
1999) \\
\end{array}$ \\
\hline 14 & Haider et,al/ 2017 & $\begin{array}{l}\text { Banglad } \\
\text { esh }\end{array}$ & $\begin{array}{l}\text { This study aims to construct an } \\
\text { index of women's autonomy to } \\
\text { analyze its effect on maternal } \\
\text { healthcare utilization in } \\
\text { Bangladesh. }\end{array}$ & Survey & $\begin{array}{l}\mathrm{n}=8753 \\
\text { women }\end{array}$ & $\begin{array}{l}\text { Bangladesh } \\
\text { Demographi } \\
\text { c and Health } \\
\text { Survey } \\
\text { (BDHS) } \\
2011\end{array}$ \\
\hline 15 & Kamiya/ 2011 & $\begin{array}{l}\text { Tajikista } \\
\mathrm{n}\end{array}$ & $\begin{array}{l}\text { Examines whether or not and how } \\
\text { women's autonomy within the } \\
\text { household affects the use of } \\
\text { reproductive health care, using a } \\
\text { household survey data from } \\
\text { Tajikistan }\end{array}$ & Survey & $\begin{array}{l}\mathrm{n}=4860 \text { women } \\
\text { from } 19 \text { to } 49 \\
\text { years old }\end{array}$ & $\begin{array}{l}\text { Tajikistan } \\
\text { Living } \\
\text { Standars } \\
\text { Survey } 2007\end{array}$ \\
\hline
\end{tabular}

Indonesian Journal Of Health Sciences Research and Development 
Amraeni, Y., \& Nirwan, M.

DOI: $10.36566 / 1 j h s r d / V o l 3 . I s s 2 / 89$

https://ijhsrd.com/index.php/ijhsrd

e- ISSN: 2715-4718

\begin{tabular}{|c|c|c|c|c|c|c|}
\hline No & $\begin{array}{c}\text { Author/year of } \\
\text { publication }\end{array}$ & Setting & Study Objectives & $\begin{array}{l}\text { Study } \\
\text { Design }\end{array}$ & Sample size & $\begin{array}{c}\text { Data } \\
\text { Source }\end{array}$ \\
\hline 16 & Haque,et.al /2012 & $\begin{array}{l}\text { Banglad } \\
\text { esh }\end{array}$ & $\begin{array}{l}\text { examine the linkage between the } \\
\text { possible influences of the extent } \\
\text { of autonomy on young mothers } \\
\text { use of reproductive health care } \\
\text { services }\end{array}$ & Survey & $\begin{array}{l}\mathrm{n}=1,778 \\
\text { currently } \\
\text { married women } \\
\text { aged } 15 \text { to } 24 \\
\text { years }\end{array}$ & $\begin{array}{l}2007 \\
\text { Bangladesh } \\
\text { Demographi } \\
\text { c Health } \\
\text { Survey } \\
\end{array}$ \\
\hline 17 & $\begin{array}{lr}\text { Hindin and } \\
\text { Muntifering/ } 2011\end{array}$ & Africa & $\begin{array}{l}\text { explored the relationships } \\
\text { between married women's } \\
\text { autonomy and the time since most } \\
\text { recent sexual intercourse in } \\
\text { Ghana, Malawi, Mali, Rwanda, } \\
\text { Uganda, and Zimbabwe }\end{array}$ & Survey & $\begin{array}{l}\mathrm{n}=1944 \text { (Ghana), } \\
\text { 5556(Malawi), } \\
5308 \text { (Mali), } \\
3927 \\
\text { (Rwanda),2812( } \\
\text { Uganda) and } \\
3594 \\
\text { (Zimbabwe) }\end{array}$ & $\begin{array}{l}\text { Demographi } \\
\text { c and Health } \\
\text { Surveys }\end{array}$ \\
\hline 18 & $\begin{array}{l}\text { Adhikar\& } \\
\text { Sawangdee/ } 2011\end{array}$ & Nepal & $\begin{array}{l}\text { Examine the factors influencing } \\
\text { infant mortality, specifically, } \\
\text { whether women's autonomy has } \\
\text { an impact on infant mortality in } \\
\text { the Nepali context }\end{array}$ & Survey & $\begin{array}{lr}\mathrm{n} & =5,545 \\
\text { children who } & \text { were born } \\
\text { were } & \text { within the five } \\
\text { years preceding } \\
\text { the survey }\end{array}$ & $\begin{array}{l}\text { Nepal } \\
\text { Demographi } \\
\text { c and Health } \\
\text { Survey, } \\
2006\end{array}$ \\
\hline 19 & $\begin{array}{l}\text { Situ.K/ } \\
2013\end{array}$ & Nepal & $\begin{array}{l}\text { Assess the association between } \\
\text { women's autonomy and maternal } \\
\text { health care utilization among } \\
\text { Nepalese women }\end{array}$ & Survey & $\begin{array}{l}\mathrm{n}=4,148 \\
\text { women }\end{array}$ & $\begin{array}{l}2011 \text { Nepal } \\
\text { Demographi } \\
\text { c Health } \\
\text { Survey }\end{array}$ \\
\hline 20 & $\begin{array}{l}\text { Tiruneh et al. } \\
\text { /2017 }\end{array}$ & Ethiopia & $\begin{array}{l}\text { assessed whether women's } \\
\text { autonomy, measured at both } \\
\text { individual and community levels, } \\
\text { is associated with maternal } \\
\text { healthcare service utilization in } \\
\text { Ethiopia }\end{array}$ & Survey & $\begin{array}{l}\mathrm{n}=14.070 \text { in } \\
2005 \text { and } \mathrm{n}= \\
16.515 \text { in } 2011 \\
\text { Women ages } \\
(15-49)\end{array}$ & $\begin{array}{l}2005 \text { and } \\
2011 \\
\text { Ethiopia } \\
\text { Demographi } \\
\text { c and Health }\end{array}$ \\
\hline 21 & $\begin{array}{l}\text { Woldemicael/ } \\
2009\end{array}$ & Eritrea & $\begin{array}{l}\text { Address some of the most } \\
\text { frequently raised questions about } \\
\text { the link between women's } \\
\text { autonomy and reproductive } \\
\text { behaviour }\end{array}$ & Survey & $\begin{array}{lr}\mathrm{n} \quad= & 8754 \\
\text { Women } & \text { ages } \\
(15-49) & \end{array}$ & $\begin{array}{l}2002 \text { Eritrea } \\
\text { Demographi } \\
\text { c and Health } \\
\text { Survey }\end{array}$ \\
\hline 22 & Widyastuti/2017 & $\begin{array}{l}\text { Indonesi } \\
\text { a } \\
\text { (Jateng) }\end{array}$ & $\begin{array}{l}\text { Examine the relationship between } \\
\text { women autonomy and ANC } \\
\text { utilization }\end{array}$ & Survey & $\begin{array}{l}\mathrm{n}=85 \text { married } \\
\text { teenage }\end{array}$ & $\begin{array}{l}\text { Primary } \\
\text { data }\end{array}$ \\
\hline
\end{tabular}

Table 2. Measurement of autonomy and associations on the impact of reproductive health

\begin{tabular}{|c|c|c|c|c|}
\hline No & $\begin{array}{l}\text { Author/year of } \\
\text { publication }\end{array}$ & Outcome & Measurement of autonomy & Association \\
\hline 1 & $\begin{array}{l}\text { Upadhyay and } \\
\text { deborah/2012 }\end{array}$ & Fertility & $\begin{array}{l}\text { Negative attitudes toward wife beating } \\
\text { and Greater household decision }\end{array}$ & $\mathrm{B}=0,5 \& 0,3$ \\
\hline 2 & $\begin{array}{l}\text { Tadesse et al/ } \\
\text { 2013. }\end{array}$ & Family Planning & $\begin{array}{l}\text { Women's attitude towards domestic } \\
\text { violence, women's involvement in } \\
\text { household decision making, and } \\
\text { exposure to sources of knowledge }\end{array}$ & $\begin{array}{l}\operatorname{AOR}((1.14,1.27) \& \\
((1.19,1.33)\end{array}$ \\
\hline 3 & $\begin{array}{l}\text { Stojanovski } \\
\text { et.al } / \mathbf{2 0 1 7}\end{array}$ & Fertility & $\begin{array}{l}\text { Labour force and inclusion in household } \\
\text { decisions }\end{array}$ & $\mathrm{RRR}=1,4, \mathrm{CI}(1.1,1.8)]$. \\
\hline 4 & $\begin{array}{l}\text { Goleen Samari } \\
\text { /2017 }\end{array}$ & Family Planning & $\begin{array}{l}\text { Household decision-making and attitudes } \\
\text { towards intimate partner violence }\end{array}$ & RRR: $1.12 \& 1.04$ \\
\hline 5 & $\begin{array}{l}\text { Renuka and } \\
\text { Jeyarathman/201 } \\
\text { 6 }\end{array}$ & Family Planning & $\begin{array}{l}\text { Decision making autonomy, movement } \\
\text { autonomy and control over economic } \\
\text { resources }\end{array}$ & $\mathrm{X} 2=11,445 \& \mathrm{X} 2=6,915$ \\
\hline
\end{tabular}


Amraeni, Y., \& Nirwan, M.

DOI: $10.36566 / 1 j h s r d / V o l 3 . I s s 2 / 89$

https://ijhsrd.com/index.php/ijhsrd

e- ISSN: 2715-4718

\begin{tabular}{|c|c|c|c|c|}
\hline No & $\begin{array}{c}\text { Author/year of } \\
\text { publication }\end{array}$ & Outcome & Measurement of autonomy & Association \\
\hline 6 & $\begin{array}{l}\text { Mosfequr } \\
\text { Rahman/2012 }\end{array}$ & Fertility & $\begin{array}{l}\text { Women's household decision-making } \\
\text { autonomy }\end{array}$ & $\begin{array}{l}\text { OR }=0,77,95 \% \text { CI: } 0,54- \\
0,88\end{array}$ \\
\hline 7 & $\begin{array}{l}\text { Patrikar,et.al/ } \\
2014\end{array}$ & Family Planning & $\begin{array}{l}\text { Women's decision-making power index } \\
\text { and women's autonomy index }\end{array}$ & $0,7 \%, 95 \%$ CI $66,2,75,28$ \\
\hline 8 & $\begin{array}{lr}\text { Abada } \& \\
\text { Tenkorang/ } 2012\end{array}$ & Fertility & $\begin{array}{l}\text { Education and wealth as indicators of } \\
\text { women's autonomy }\end{array}$ & OR $0.949(0.150)$ \\
\hline 9 & Blackstone/ 2017 & Family Planning & $\begin{array}{l}\text { Attitudes towards intimate partner } \\
\text { violence and decision-making }\end{array}$ & $\beta=0,092 ; p<0,05$ \\
\hline $\mathbf{1 0}$ & Ghose et.al/2017 & $\begin{array}{ll}\text { Health } & \text { Care } \\
\text { Utilization } & \end{array}$ & $\begin{array}{l}\text { Decision-making status on respondent's } \\
\text { own healthcare, large household } \\
\text { purchases, having a say on child's } \\
\text { healthcare and visiting to family or } \\
\text { relatives }\end{array}$ & $95 \%$ CI 0,794 - 1,799 \\
\hline 11 & $\begin{array}{l}\text { Woldemicael/ } \\
\mathbf{2 0 0 7}\end{array}$ & $\begin{array}{l}\text { Health } \\
\text { Utilization }\end{array}$ & $\begin{array}{l}\text { Women's decision-making power, } \\
\text { freedom of movement and women's } \\
\text { attitude toward partner's violence }\end{array}$ & OR $0,56-1,60$ \\
\hline 12 & $\begin{array}{l}\text { Furuta } \\
\text { and } \\
\text { Salway/2006, }\end{array}$ & $\begin{array}{l}\text { Health } \\
\text { Utilization }\end{array}$ & $\begin{array}{l}\text { Household-decision } \\
\text { employment and influence over earning, } \\
\text { and spousal discussion of family } \\
\text { planning }\end{array}$ & OR $1,4 \& 1,3$ \\
\hline 13 & Mistry et,al/2009 & $\begin{array}{l}\text { Health } \\
\text { Utilization }\end{array}$ & $\begin{array}{l}\text { Measured in the } 3 \text { dimensions of } \\
\text { decision-making autonomy, permission } \\
\text { to go out, and financial autonomy }\end{array}$ & OR $1,05,1,08 \& 1,111$ \\
\hline 14 & $\begin{array}{l}\text { Haider et,al/ } \\
2017\end{array}$ & $\begin{array}{l}\text { Health } \\
\text { Utilization }\end{array}$ & $\begin{array}{l}\text { decision making regarding healthcare, } \\
\text { financial autonomy and freedom of } \\
\text { movement }\end{array}$ & \\
\hline 15 & Kamiya/ 2011 & $\begin{array}{l}\text { Health } \\
\text { Utilization }\end{array}$ & $\begin{array}{l}\text { Womens decision on child's well being, } \\
\text { on buying major items and on borrowing } \\
\text { money }\end{array}$ & $3,6 \%$ dan $3,0 \%$ \\
\hline 16 & Haque,et.al /2012 & $\begin{array}{l}\text { Health } \\
\text { Utilization }\end{array}$ & $\begin{array}{l}\text { Dimensi otonomi dengan Employment } \\
\text { and economic decision making }\end{array}$ & $\begin{array}{l}[\mathrm{AOR}], \quad 1.64 ; 95 \% \mathrm{CI}], \\
1.17-2.23\end{array}$ \\
\hline 17 & $\begin{array}{l}\text { Hindin and } \\
\text { Muntifering/ } \\
\mathbf{2 0 1 1}\end{array}$ & Fertility & Household decision making & HR 0,90 \\
\hline 18 & $\begin{array}{l}\text { Adhikar\& } \\
\text { Sawangdee/ } 2011\end{array}$ & Fertility & $\begin{array}{l}\text { Literacy status, decision on own health } \\
\text { care, on making large household } \\
\text { purchases, household purchases for daily } \\
\text { nees, and visits to family or relatives }\end{array}$ & $\mathrm{OR}=0,61 \& 0,74$ \\
\hline 19 & $\begin{array}{l}\text { Situ.K/ } \\
2013\end{array}$ & $\begin{array}{l}\text { Health } \\
\text { Utilization }\end{array}$ & $\begin{array}{l}\text { Decision making regarding health care, } \\
\text { large household purchases, visiting } \\
\text { friends or relatives and spending money } \\
\text { earned by husbands }\end{array}$ & $\begin{array}{l}\text { OR }=1,69,95 \% \quad \mathrm{CI}= \\
1,41-2,03\end{array}$ \\
\hline 20 & $\begin{array}{l}\text { Tiruneh et al. } \\
/ \mathbf{2 0 1 7}\end{array}$ & $\begin{array}{l}\text { Health } \\
\text { Utilization }\end{array}$ & $\begin{array}{l}\text { Decision making power on household } \\
\text { and attitude toward wife beating }\end{array}$ & $\begin{array}{l}\mathrm{AOR}=1,16 ; 95 \% \mathrm{CI}= \\
1,08-1,90\end{array}$ \\
\hline 21 & $\begin{array}{l}\text { Woldemicael/ } \\
\mathbf{2 0 0 9}\end{array}$ & Fertility & $\begin{array}{l}\text { Women agree or disagree with negative } \\
\text { gender norms (i.e. wife } \\
\text { Beating }\end{array}$ & NA \\
\hline 22 & Widyastuti/2017 & $\begin{array}{l}\text { Health } \\
\text { Utilization }\end{array}$ & Participation on decision making & $\begin{array}{l}\text { (p-value }=0.013, X 2= \\
6.168\end{array}$ \\
\hline
\end{tabular}




\section{Utilization of services}

Review studies show the dominance of autonomy related to the utilization of health services. ten studies conducted research to identify the relationship between autonomy and utilization of services, both for maternal health and for children. Eight studies were conducted in South Asian countries (Bangladesh, Nepal, India) and two others in African countries. Ghose (2017) shows the association between participation in decision making and the utilization of maternal health services for Bangladeshi women. The results showed that, compared to women who could make decisions, women in urban areas who had decided on their health care with their husbands / partners had 20\% (95\% CI 0.794 to 1,799$)$ more likely to attend at least four antenatal visits and they settling in rural areas has $35 \%$ (95\% CI 0.464 to 0.897 ) lower chances of attending at least four antenatal visits.

This is supported by Haider (2017) which shows that every one increase in autonomy score, the utilization of maternal health services will increase by 0.14 for ANC, and 0.13 for PNC. Research in the same country was also carried out by Haque (2012), but with young female respondents (15-24 years) showing that young women who have a higher degree of autonomy are more likely to receive adequate ANC. Likewise, women with moderate autonomy were 1.40 times more likely to give birth assisted by trained personnel than women with low autonomy. Widyastuti (2017) also with the subject of Indonesian adolescents reveals that it is important for adolescents to have decision-making power in the family, especially during pregnancy.

Studies in another South Asian country in Nepal also showed that women with autonomy in health care were significantly more likely to attend $\mathrm{ANC}=3$ visits $(\mathrm{OR}=$ $1.69,95 \% \mathrm{CI}=1.41-2.03)$ and had the opportunity to give birth in a health facility ( $\mathrm{OR}=1.4495 \% \mathrm{CI}=1.26-1.64) 31$. African countries such as Eritrea and Ethiopia also express the same thing that the strong positive influence of a woman's decision making enables them to participate in decisions. The percentage of women with high decision-making power has a positive relationship with antenatal care visits 36 . Furuta's (2006) study is slightly different from the others, because the results showed that although the relationship was not consistent across all indicators, husband and wife discussions about family planning were associated with an increased likelihood of receiving antenatal care and childbirth..

\section{Family Planning}

Blackstone (2017) in his study in African countries (Ghana) and regression analysis showed that women who are abused have a low chance to use contraception. In other African countries (Ethiopia) studies related to family planning autonomy associated with dimensions in household decisions and attitudes toward violence with the results showed there is a correlation between the two34. Renuka (2016) in Coimbatore also proves the alleged relationship with chi-square analysis which shows a strong relationship between decision-making power and contraceptive use and between indirect dimensions and educational indicators that have a strong relationship with contraceptive use.

Samari (2017) in Egypt found that women with a high degree of autonomy tend to use the LARC method compared to shortacting ones. The same thing with research Patrikar (2014) in India found evidence that women with low levels of decision making has a higher degree of autonomy that allows the use of contraception.

\section{Fertility}

Uphadhyay (2012) conducted a study in Guinea and Zambia, which is an African state, identify negative attitudes violent relationship with the ideal number of children fewer. Rahman (2012) conducted a 
study in Bangladesh showing that women with a high autonomy scale of unwanted pregnancies were around 23\%. Stojanovski (2017) also conducted research in roma which showed that women who are able to make decisions showed 1.4 times control unwanted pregnancy.

In another South Asian country, Nepal has shown a study that women who are literate are 39 percent less likely to die of infants than illiterate women. Likewise, women who are able to participate in decision making have a 26 percent lower likelihood of infant mortality than those who are not involved in the decision-making process4.

\section{DISCUSSION}

the concept of autonomy does not only concern social issues, but also develops in health sciences. The autonomy of women in making decisions, especially in terms of reproductive health, is very important for better maternal and child health18. From various factors that hinder women's access to reproductive health in developing countries, it proves that women's autonomy has an important role10.

The ability of women to participate in health decisions depends in part on autonomy13. People with low income have an impact on the status of women with limited autonomy and the ability to make decisions in every aspect of life 30 . This illustrates that a society with such conditions still has a strong social structure that rigidly defines the roles of men and women, usually coded in religious, ethnic and social traditions 14.

R. Freedman in the 2012 IDHS Further Analysis, describes a model between fertility and social conditions with the prevailing norms. The results of further analysis of the 2012 IDHS show that social structures and norms have a significant effect on fertility. Previous research, located in Sehore, a district of Madhya Pradesh in India by Char et al (2010), showed that family and prevailing norms influence women to make decisions regarding the number of children and the use of contraception. According to Freedman, variables that directly affect fertility are basically also influenced by the norms prevailing in society.

In making decisions related to fertility, the relationship between family members is influenced, especially the relationship between husband and wife. Husband and wife relations are reflected in gender relations which are the construction of sociocultural values and norms at the community level2. The success of the Family Planning (KB) program is highly dependent on community participation. It is hoped that the high level of community participation in the family planning program can achieve the goals of the family planning program. One indicator of the success of the Family Planning $(\mathrm{KB})$ program is the declining rate of population growth. Women's participation and empowerment in family planning programs is a potential strategy in increasing women's access and cultural transformation8.

Women's participation in the family planning program supports women's empowerment in making decisions for themselves and their families22. The sociocultural context that describes the relationship between women's characteristics at the individual level in decision-making and autonomy is the main mediator intervention between women's status and reproductive outcomes 19 . Gender inequality affects communication, thereby limiting women's decision making to access reproductive health3.

Reproductive health program and policy opportunities in developing countries are basically related to the integration of women's empowerment into health programs. Based on the literature study, the program strategy offered relates to contraceptive use through the promotion of women's empowerment, namely decision making and education. thus impacting women's ability to negotiate fertility decisions. Meanwhile, for the utilization of 
health services, the government can focus on individual awareness and public awareness of women's rights to use maternal and child health services. Other policies such as the establishment of socioeconomic improvement programs for women and education programs to increase the status and power of women in the household and society can effectively increase the utilization of women's health and health services.

Based on the results of a study in India recommends a home visit strategy of village health workers through the National Rural Health Program, Accreditation of Social Health Accreditation, which can promote women's autonomy and utilization of maternity care services that include prenatal care, childbirth and postnatal care. Similar to studies in Bangladesh, the results of the study emphasize the importance of focusing on a comprehensive strategy to increase women's autonomy rather than just focus on the socio-economic improvement. In addition, intervention programs in improving health service is expected to pay attention to the socio-cultural elements as a guideline. Meanwhile, the education factor is highly emphasized in Nepal to increase women's autonomy which can directly increase the use of health services and women's ability to regulate fertility.

\section{CONCLUSION}

This review identifies gaps from various studies in developing countries related to reproductive health impact data in relation to women's autonomy. And in fact the results of the study indicate an association between the two. The use of the autonomy dimension to measure associations is still not perfect because some literature uses a single dimension or a combination of two dimensions.

\section{REFERENCES}

1. Abada Teresa \& EricTenkorang. Women's Autonomy and Unintended Pregnancies in The Philippines. 2012.J. Biosoc. Sci., 44, 703-718. doi:10.1017/S0021932012000120.

2. Abdullah Irwan. Penelitian Berwawasan Gender Dalam Ilmu Sosial. 2003.Humaniora Volume XV, No.3. Universitas Gadjah Mada.

3. Acharya, Dev R, Bell, Jacqueline S, Simkhada, Padam, Teijlingen, Edwin R Van, Regmi, \&Pramod R.Research Women's autonomy in household decision-making: a demographic study in Nepal.2010. journal of Reproductive Health, 7(15);1-12. http://www.reproductive-healthjournal.com/content/7/1/15.

4. Adhikari Ramesh \&Yothin Sawangdee. Influence of women's autonomy on infant mortality in Nepal.2011. Reproductive Health, 8:7. http://www.reproductive-healthjournal.com/content/8/1/7.

5. Basu AM.Culture, the Status of Women, and Demographic Behaviour: Illustrated with the case of India. 1992.Oxford, UK: Clarendon Press.

6. Blackstone Sarah. Women's Empowerment, Household Status and ContraceptionUse in Ghana.2012.J. Biosoc. Sci. 49, 423-434. doi:10.1017/S0021932016000377.

7. Bloom SS, Wypij D, Das Gupta M. Dimensions of women's autonomy and the influence on maternal health care utilization in a north Indian city. Demography. 2001 Feb;38(1):67-78. doi: 10.1353/dem.2001.0001. PMID: 11227846.

8. Bongaarts, John \& Sinding, Steven. A Response to Critics of Family Planning Programs. International perspectives on 
sexual and reproductive health. 2009. 35. 39-44. 10.1363/ifpp.35.039.09.

9. Char, Arundhati \& Saavala, Minna \& Kulmala, Teija. Influence of mothers-inlaw on young couples' family planning decisions in India. 2010.Reproductive health matters. 18. 154-62. 10.1016/S0968-8080(10)35497-8.

10. Do, Mai \& Kurimoto, Nami. Women's Empowerment and Choice of Contraceptive Methods in Selected African Countries. 2012.International perspectives on sexual and reproductive health. 38. 23-33. 10.1363/3802312.

11. Dyson, T., \& Moore, M. On kinship structure, female autonomy, and demographic behavior in India. 1983. Population and Development Review, 9, $35-60$.

12. Furuta M, Salway S. Women's position within the household as a determinant of maternal health care use in Nepal. IntFam Plan Perspect. 2006 Mar;32(1):17-27. doi: 10.1363/3201706. PMID: 16723298.

13. Ghose Bishwajit, Da feng, Shangfeng Tang, Sanni Yaya, Zhifei He, Ogochukwu Udenigwe, Sharmistha Ghosh, Zhanchun Feng. Women's decision-making autonomy and utilisation of maternal healthcare services: results from the Bangladesh Demographic and Health Survey.2017.BMJ Open;7:e017142. doi:10.1136/ bmjopen-2017-017142

14. Osamor, Pauline \& Grady, Christine. Women's autonomy in health care decision-making in developing countries: A synthesis of the literature. 2016. International Journal of Women's Health. 8. 191. 10.2147/IJWH.S105483.

15. Haider Rifat Mohammad, Zaina P.Qureshi \& M.Mahmud Khan.Effects of women's autonomy on maternal healthcare utilization in Bangladesh: Evidence from a national survey.2017.
Sexual \& Reproductive Healthcare $14.40-47$

.https://doi.org/10.1016/j.srhc.2017.09.0 02.

16. Haque SE, Rahman M, Mostofa MG, Zahan MS. Reproductive health care utilization among young mothers in Bangladesh: does autonomy matter?.2012.Womens Health Issues.;22(2):e171-e80

17. Hindin, Michelle. Women's autonomy, women's status and fertility-related behavior in Zimbabwe.(2000). Population Research and Policy Review. 19. 255-282. 10.1023/A:1026590717779.

18. International Conference on Population and Development (1994). [http:/ /www.un.org/ecosocdev/geninfo/populat in/icpd.htm]. accessed on 14/ 09/2018

19. Jejeebhoy, S. J., \& Sathar, Z. A. Women's Autonomy in India and Pakistan: The Influence of Religion and Region. . (2001)Population and Development Review, 27, 687-712. http://dx.doi.org/10.1111/j.17284457.2001.00687.x.

20. Kabeer N. Resources, agency, achievements: reflections on the measurement of women's empowerment. (1999).Dev Change.30(3):435464.https://doi.org/10.1111/14677660.00125

21. Kamiya Yusuke. Women's autonomy and reproductive health care utilisation. epirical evidence from Tajikistan. (2011) Health Policy 102 (2011) 304-313 dx.doi.org/10.1016/j.healthpol.2011.04.0 01 .

22. Kinfu Yohannes and Monika Sawhney. Effect of women's empowerment and socio-economic status on choice and use of family planning methods in SubSaharan Africa. 2011. UAPS. 
23. Kishor S, Subaiya L,. Understanding women's empowerment: a comparative analysis of demographic and health surveys data. (2005).DHS comparative reports no.20. Calverton, MD: Macro International.

24. Mistry Ritesh, Osman Galal \& Michael LuWomen's autonomy and pregnancy care in rural India. Social Science and Medicine.. (2009). 926-933. doi:10.1016/j.socscimed.2009.07.008

25. Mullany, B. C., Hindin, M. J., \& Becker, S.Can women's autonomy impede male involvement in pregnancy health in Katmandu, Nepal?(2005) Social Science and Medicine, 61(9), 1993-2006. https://doi.org/10.1016/j.socscimed.2005 .04.006.

26. Patrikar, Basannar, and Maj Seema Sharma. Women empowerment and use of contraception. Medical Journal Armed Forces India. (2014) 70. 253256.

http://dx.doi.org/10.101016/j.mjafi.2013 .12 .014 .

27. Rahman Mosfequr. Women's autonomy and Unintended pregnancy among currently pregnant women in Bangladesh. Matern Child Health J (2012) 16:1206-1214. DOI 10.1007/s10995-011-0897-3

28. Renuka \& Jeyarathman.. Women's Autonomy and Family Planning Practices among Married Women in Coimbatore. Asian J. Nursing Edu. and Research 6(2): April- June 2016. DOI: 10.5958/2349-2996.2016.00039.2.

29. Samari Goleen. Women's empowerment and short- and longacting contraceptive method use in Egypt.Culture, Health \& Sexuality.(2017) ISSN: 1369-1058 (Print) 1464-5351. DOI: 10.1080/13691058.2017.1356938

30. Senarath, Upul \& Gunawardena, Nalika. Women's Autonomy in Decision Making for Health Care in South Asia.
(2009)Asia-Pacific journal of public health / Asia-Pacific Academic Consortium for Public Health. 21. 13743. 10.1177/1010539509331590.

31. Situ.K.C. Women's Autonomy And Maternal Health Care Utilization In Nepal. 2013. University of Tampere. School of Health Sciences (Public Health).

32. Sharan Mona, Saifudin Ahmed \& Donna Strobino. Influence of Women's Autonomy and Access to Health Services on Maternal Health Care Utilization in Rural India. 2017. Department of Population and Family Health Sciences. Johns Hopkins Bloomberg School of Public Health.

33. Stojanovski Kriesfer, Teresa Janevic, Blasko Kasapinov, Zeljka Stamenkovic \& Janko Jankovic. An Assesment ofRomani Women's Autonomy and Timing of Pregnancy in Serbia and Macedonia. Matern Child Health J (2017) 21:1814-1820. DOI 10.1007/s10995-017-2292-1.

34. Tadesse, Mekonnen \& Teklie, Habtamu \& Yazew, Gorfu \& Gebreselassie, Tesfayi. Women's Empowerment as a Determinant of Contraceptive Use in Ethiopia. Further Analysis of the 2011 Ethiopia Demographic and Health Survey.

35. Demographic and Health Survey (2017). DHS Further Analysis Reports No. 82.Calverton, Maryland, USA: ICF International.

36. Tiruneh Fentanesh Nibret, Kun-Yang Chuang \& Ying-Chih Chuang. Women's autonomy and maternal healthcare service utilization in Ethiopia. BMC Health Services Research (2017) 17:718. DOI 10.1186/s12913-017-26709

37. Upadhyay UD, Karasek D. Women's empowerment and ideal family size: an examination of DHS empowerment 
Amraeni, Y., \& Nirwan, M.

DOI: 10.36566/ijhsrd/Vol3.Iss2/89

https://ijhsrd.com/index.php/ijhsrd

measures in sub-Saharan Africa.(2017)

Int Perspect Sex Reprod Health.;38:78-

89.

38. Widyastuti Whinda. Otonomi Wanita dan Pemanfaatan Antenatal Care (ANC) pada Primigravida Remaja di Daerah Pedesaan, Jawa Tengah. Indonesian Journal of Nursing Practices Vol.1.No 2Juni 2017. DOI Number : 10.18196/ijnp. 1258 .

39. Woldemicael, G.Do women with higher autonomy seek more maternal and child health-care? Evidence from Ethiopia and Eritrea. , 2007. 49(1), hal.1-27. Available at: http://www.demogr.mpg.de.

40. Woldemicael G\& Tenkorang EY. Women's autonomy and maternal health-seeking behavior in Ethiopia. 2009. Maternal and child health journal.;14(6):988-98.doi: 10.1007/s10995-009-0535-5. 